\title{
Gradual Clamping Reduced Ischemia-Reperfusion Injury in an Isolated Rat Heart Model
}

\author{
Hongbin Feng1, Hongli Wang2, Yang Zhao1, Zhinan Zheng1, Sanqing Jin ${ }^{1 *}$ \\ ${ }^{1}$ Department of Anesthesia, The Sixth Affiliated Hospital, Sun Yat-sen University, Guangzhou, China \\ ${ }^{2}$ Department of Anesthesia, Tianjin Fifth Central Hospital, Tianjin, China \\ Email: "sanqingin@hotmail.com
}

Received 9 May 2016; accepted 14 June 2016; published 17 June 2016

Copyright (C) 2016 by authors and Scientific Research Publishing Inc.

This work is licensed under the Creative Commons Attribution International License (CC BY).

http://creativecommons.org/licenses/by/4.0/

(c) (i) Open Access

\begin{abstract}
Objectives: We hypothesized that the organisms and their organs or tissues could adapt themselves to the gradual changes of environment for surviving or reducing damage. This study explored whether gradual clamping (GC) could reduce myocardial ischemia-reperfusion (IR) injury in rat heart. Methods: Twelve rats were randomized to IR group and GC group, then the hearts were isolated and perfused with Langendorff apparatus. Before cardioplegia, the perfusion was stopped abruptly in IR group while slowly with 5-minute in GC group. The hearts were subjected to 30-minute ischemia and 60-minute reperfusion. The left ventricular develop pressure (LVDP) and systolic pressure (LVSP), the maximal rate of the increase and decrease of left ventricular pressure $\left(+d p / d t_{\max },-d p / d t_{\max }\right)$ were measured by polygraph system at different time points. The recovery of the variables was expressed as the ratio of these values at individual time point after reperfusion to the baseline respectively. Results: The recovery of LVDP after reperfusion was better than that in IR group $(P=0.034)$. No significant difference in the recovery of $L V S P,+d p / d_{\max }$ and $-\mathrm{dp} / \mathrm{dt}_{\max }$ between groups was observed. Conclusions: Gradual clamping could improve the recovery of LVDP after IR, suggesting that gradual clamping could reduce myocardial IR injury.
\end{abstract}

\section{Keywords}

Gradual Clamping, Ischemia-Reperfusion Injury, Gradual Adaptation, Rat Heart Model

\section{Introduction}

Cardiopulmonary bypass (CPB), considered as one of the most important clinical advances in medicine in the *Corresponding author. 
1950s, has been widely applied in cardiac surgery [1]. However, CPB can cause myocardial ischemia-reperfusion (IR) injury which is closely associated with postoperative complications such as myocardial infarction and cardiac failure [2] [3]. Although ischemic preconditioning (IPC) and remote ischemic preconditioning (RIPC) have the potential to attenuate IR injury [4] [5], their protective effects in clinic are still controversial and limited [6]-[8]. Therefore, more protective strategies need to be explored.

According to our clinical experience, some severe bleeding patients with extremely low hemoglobin could be rescued successfully and recovered without any clinical complications. Sanqing Jin brought up the hypothesis of gradual adaptation, meaning that the organisms or the human body or their organs and tissues could change their own innate ongoing normal process to adapt to the gradual changes of environment for surviving or reducing damage, including the changes from one state to another state, either from physiologic status to pathologic status or pathologic status to physiologic status. According to the above hypothesis, we further hypothesized that gradual clamping, which means that the perfusion of the heart is blocked gradually at a constant speed till to cessation of the perfusion, but not ceased abruptly, could induce the myocardial gradual adaptation to ischemia, and so could reduce the myocardial ischemia-reperfusion injury.

This study aimed to explore whether gradual clamping could reduce myocardial IR injury in isolated Langendorff-perfused rat heart.

\section{Materials and Methods}

\subsection{Ethics Committee Approval}

The study was approved by the Animal Care committee of Sun Yet-sen University (IACUC-2012-0802) and conformed to the guidelines on animal welfare of the National Committee for Animals Experiments.

\subsection{Animals}

The experiments were performed on twelve hearts harvested from 12 male Sparage-Dawley rats weighing from $300 \mathrm{~g}$ to $350 \mathrm{~g}$ (10 weeks to 12 weeks old) obtained from Medical Experimental Animal Center of Guangdong Province, Guangzhou, China. Rats were randomly assigned to two groups ( $\mathrm{n}=6$ rats for each group): ischemia reperfusion group (IR group) and gradual champing group (GC group). Animals were fed with normal rat chow in specific pathogen free (SPF) laboratory. After one week of adaptation, body weight was measured and recorded, rats entered into the experiment according to the grouping.

\subsection{Experimental Protocol}

The rats were heparinized by heparin $1000 \mathrm{IU} / \mathrm{kg}$ intraperitoneally, and 25 minutes later they were anesthetized with intraperitoneal sodium pentobarbital $(60 \mathrm{mg} / \mathrm{kg}$, Shanghai Chemical Reagent Procurement and Supply Station, China). After anesthesia, the rats were sacrificed by cervical dislocation, rapid chest opening was performed, then hearts of the rats were quickly removed, and blood in the hearts was washed away in Krebs-Henseleit bicarbonate buffer of $4^{\circ} \mathrm{C}$. The aorta of the heart was rapidly cannulated and the heart was fixed to the Langendorff apparatus (AD Instruments, Australia) and perfused by Krebs-Henseleit bicarbonate buffer [11.0 mmol/l glucose, $118.5 \mathrm{mmol} / \mathrm{l} \mathrm{NaCl}, 4.75 \mathrm{mmol} / \mathrm{l} \mathrm{KCl}, 1.19 \mathrm{mmol} / \mathrm{l} \mathrm{MgSO}$, $1.18 \mathrm{mmol} / \mathrm{KH}_{2} \mathrm{PO}_{4}, 25.0 \mathrm{mmol} / \mathrm{l} \mathrm{NaHCO}$, and $\left.1.4 \mathrm{mmol} / \mathrm{l} \mathrm{CaCl}_{2}\right]$ at $\mathrm{pH}$ 7.4. The buffer was bubbled with $95 \% \mathrm{O}_{2}$ and $5 \% \mathrm{CO}_{2}$ at $37^{\circ} \mathrm{C}$. The time from chest opening to perfusion by Langendorff apparatus was controlled less than 2 minutes. All the reagents mentioned above and below were purchased from Guangzhou Chemical Reagent Factory (China) unless specially noted.

A latex balloon was introduced into the left ventricle from left atrium after the recovery of heart beat. The heart was perfused at a constant flow rate of $11 \mathrm{ml} / \mathrm{min}$ for 30 minutes for equilibrium. At 10 minutes of equilibrium, the balloon was expanded with water to achieve a physiological end-diastolic pressure of 4 - $8 \mathrm{mmHg}$. A pressure transducer (AD Instruments Ltd, Australia) was connected to the balloon by a rigid thin tube, and the transducer was attached to a bridge amplifier (AD Instruments Ltd., Australia) before connecting to the Power Lab biosignal collecting system (AD Instruments Ltd., Australia).

And then two different protocols were performed depending on the grouping. After equilibrium, the perfusion of IR group was stopped abruptly while the perfusion of the GC group was diminished to cessation by a constant speed with 5 minutes, and this procedure was achieved by slowly adjusting the flow button in the Langendorff apparatus manually performed by the skillfully trained doctor. Then $5 \mathrm{ml} 4^{\circ} \mathrm{C}$ St Thomas' II cardioplegic solu- 
tion [147 mmol/l NaCl, $20 \mathrm{mmol} / \mathrm{l} \mathrm{KCl}, 16 \mathrm{mmol} / \mathrm{l} \mathrm{MgCl}_{2}, 2 \mathrm{mmol} / \mathrm{l} \mathrm{CaCl}$ and $1 \mathrm{mmol} / \mathrm{l}$ procaine] was injected to the hearts of both groups through aortic root immediately after the perfusion was totally stopped. The hearts were then subjected to global ischemia for 30 minutes at $30^{\circ} \mathrm{C}$ and reperfusion for 60 minutes at a constant flow rate of $11 \mathrm{ml} / \mathrm{min}$ at $37^{\circ} \mathrm{C}$.

\subsection{Observed Variables}

The Power Lab biosignal collecting system (AD Instruments Ltd, Australia) and the software of Lab Chart 7 (AD Instruments Ltd., Australia) were used to record and calculate the left ventricular systolic pressure (LVSP), left ventricular develop pressure (LVDP), the maximal rate of the decrease of left ventricular pressure $\left(-\mathrm{dp} / \mathrm{dt}_{\max }\right)$ and the maximal rate of the increase of left ventricular pressure $\left(+\mathrm{dp} / \mathrm{dt}_{\max }\right)$ at the end of equilibrium period (baseline, $\left.T_{1}\right), 5$ minutes $\left(T_{2}\right), 10$ minutes $\left(T_{3}\right), 20$ minutes $\left(T_{4}\right), 30$ minutes $\left(T_{5}\right), 40$ minutes $\left(T_{6}\right), 50$ minutes $\left(\mathrm{T}_{7}\right), 60$ minutes $\left(\mathrm{T}_{8}\right)$ after reperfusion.

\subsection{Statistical Analysis}

The recovery of LVDP, LVSP, $+\mathrm{dp} / \mathrm{dt}_{\max }$ and $-\mathrm{dp} / \mathrm{dt}_{\max }$ after reperfusion was expressed as the ratio of the value at time points $\left(T_{2}, T_{3}, T_{4}, T_{5}, T_{6}, T_{7}, T_{8}\right)$ after reperfusion to the value at baseline $\left(T_{1}\right)$ respectively. Data were described as mean \pm standard deviation of the mean (SD) and analyzed by repeated-measures analysis of variance with SPSS 16.0 statistical software package. Statistical significances were evaluated at a two-tailed significance level of 0.05 .

\section{Results}

\subsection{Recovery of LVDP}

The changing trend of LVDP in GC group was different from that in IR group, and recovery of LVDP after reperfusion was better than that in IR group $(P=0.034)$. Besides, LVDP varied with time $(P=0.000)$ : compared to the baseline $\left(\mathrm{T}_{1}\right)$, LVDP decreased after reperfusion and reached significant differences at $\mathrm{T}_{2}(P=0.002)$ and $\mathrm{T}_{3}(P=0.013)$, and then gradually increased and reached significant differences at $\mathrm{T}_{6}(P=0.012), \mathrm{T}_{7}(P=0.020)$ and $\mathrm{T}_{8}(P=0.043)$. But there was no interaction between the time factor and the grouping factor $(P>0.05)$, so we could compare neither the difference of LVDP between groups at an individual time point, nor the difference between the individual time point to baseline in only one group (Figure 1).

\subsection{Recovery of LVSP}

The changing trends of LVSP in two groups did not achieve statistical significance $(P=0.190)$. LVSP varied with time $(P=0.000)$ : compared to the baseline $\left(T_{1}\right)$, LVSP decreased after reperfusion and reached significant differences at $\mathrm{T}_{2}(P=0.002)$ and $\mathrm{T}_{3}(P=0.029)$. But there was no interaction between the time factor and the

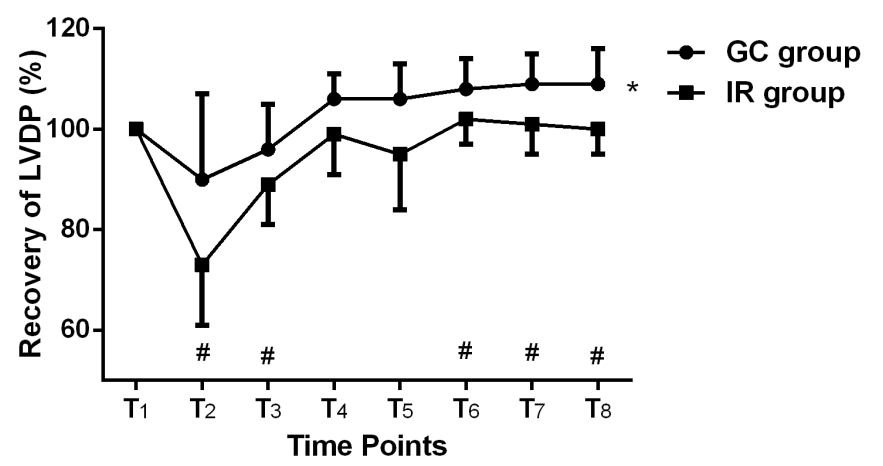

Figure 1. The recovery of LVDP between GC group and IR group. The recovery of the LVDP was expressed as the ratio of LVDP at individual time point after reperfusion to the baseline respectively. The letters $T_{1}, T_{2}, T_{3}, T_{4}, T_{5}, T_{6}, T_{7}$ and $T_{8}$ on the abscissa represent the end of equilibrium period (baseline), $5 \mathrm{~min}, 10 \mathrm{~min}, 20 \mathrm{~min}, 30 \mathrm{~min}, 40 \mathrm{~min}, 50 \mathrm{~min}$ and $60 \mathrm{~min}$ after reperfusion, respectively, same as in Figures $2-4$. Mean \pm SD. $n=6$ in each group. ${ }^{*} P<0.05$ vs IR group; ${ }^{\#} P<0.05$ vs baseline $\left(\mathrm{T}_{1}\right)$. 
grouping factor $(P>0.05)$, so we could not compare the difference between the individual time point to baseline in only one group (Figure 2).

\subsection{Recovery of $+\mathrm{dp} / \mathrm{dt}_{\max }$}

The changing trends of $+\mathrm{dp}_{\mathrm{dt}} \mathrm{dt}_{\max }$ in two groups did not achieve statistical significance $(P=0.116)$. The $+\mathrm{dp} / \mathrm{dt}_{\max }$ varied with time $(P=0.000)$ : compared to the baseline $\left(\mathrm{T}_{1}\right),+\mathrm{dp} / \mathrm{dt}_{\max }$ decreased after reperfusion and reached significant differences at $\mathrm{T}_{2}(P=0.002)$ and $\mathrm{T}_{3}(P=0.009)$, and then gradually increased and reached significant differences at $\mathrm{T}_{6}(P=0.025), \mathrm{T}_{7}(P=0.009)$ and $\mathrm{T}_{8}(P=0.022)$. But there was no interaction between the time factor and the grouping factor $(P>0.05)$, so we could not compare the difference between the individual time point to baseline in only one group (Figure 3).

\subsection{Recovery of $-\mathrm{dp} / \mathrm{dt}_{\max }$}

The changing trends of $-\mathrm{dp} / \mathrm{dt}_{\max }$ in two groups did not achieve statistical significance $(P=0.075)$. The $-\mathrm{dp} / \mathrm{dt}_{\max }$ varied with time (time factor $P=0.000)$ : compared to the baseline $\left(\mathrm{T}_{1}\right),-\mathrm{dp} / \mathrm{dt}_{\max }$ decreased after reperfusion and reached significant differences at $\mathrm{T}_{2}(P=0.000), \mathrm{T}_{3}(P=0.000)$ and $\mathrm{T}_{4}(P=0.003)$. But there was no interaction between the time factor and the grouping factor $(P>0.05)$, so we could not compare the difference between the individual time point to baseline in only one group (Figure 4).

\section{Discussion}

The present study showed that gradual clamping could improve the recovery of LVDP, demonstrating that gradual clamping could improve left ventricular systolic function recovery after myocardial IR. This result initially verified our novel hypothesis of gradual adaptation in the aspect of myocardial IR injury.

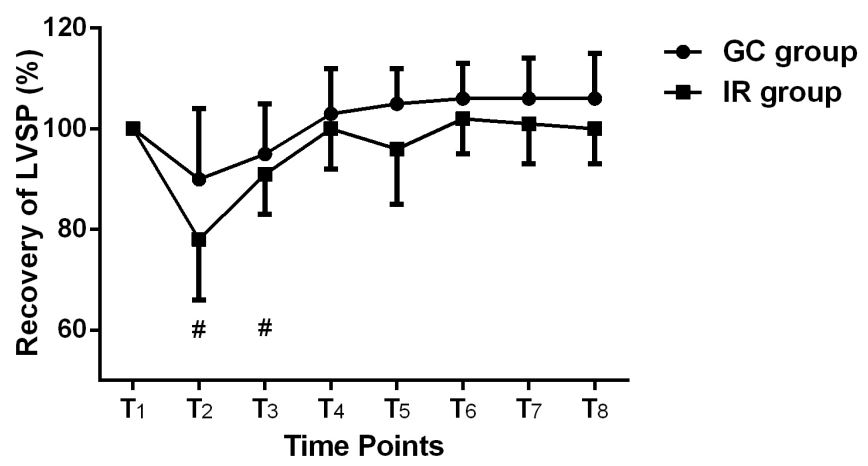

Figure 2. The recovery of LVSP between GC group and IR group. The recovery of the LVSP was expressed as the ratio of LVSP at individual time point after reperfusion to the baseline respectively. The recovery of LVSP had no significant difference between 2 groups $(P=0.190)$. Mean \pm SD. $n=6$ in each group. ${ }^{\#} P<0.05$ vs baseline $\left(T_{1}\right)$.

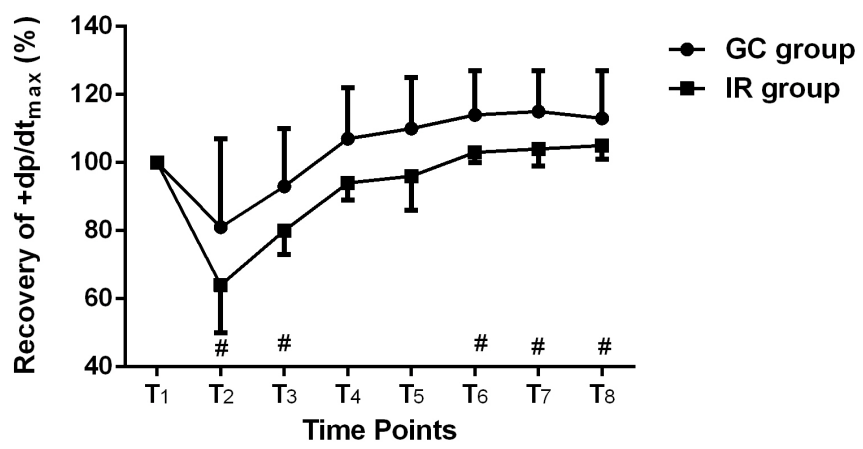

Figure 3. The recovery of $+\mathrm{dp} / \mathrm{dt}_{\max }$ between GC group and IR group. The recovery of the $+\mathrm{dp} / \mathrm{dt}_{\max }$ was expressed as the ratio of $+\mathrm{dp} / \mathrm{dt}_{\max }$ at individual time point after reperfusion to the baseline respectively. The recovery of $+\mathrm{dp}_{\mathrm{dt}}$ max $\mathrm{had}$ no significant difference between the 2 groups $(P=0.116)$. Mean \pm SD. $n=6$ in each group. ${ }^{\#} P<0.05$ vs baseline $\left(T_{1}\right)$. 


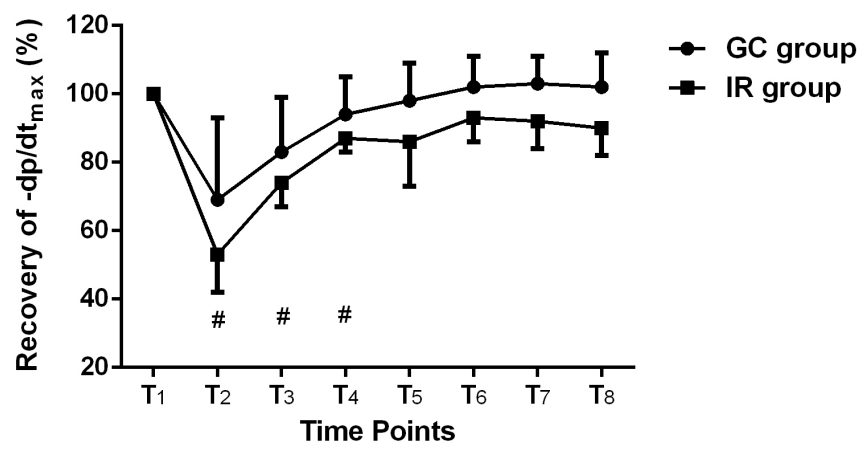

Figure 4. The recovery of $-\mathrm{dp} / \mathrm{dt}_{\max }$ between GC group and IR group. The recovery of the $-\mathrm{dp} / \mathrm{dt}_{\max }$ was expressed as the ratio of $-\mathrm{dp} / \mathrm{dt}_{\max }$ at individual time point after reperfusion to the baseline respectively. The recovery of $-\mathrm{dp} / \mathrm{dt}_{\max }$ had no significant difference between the 2 groups $(P=0.075)$. Mean \pm SD. $n=6$ in each group. ${ }^{\#} P<0.05$ vs baseline $\left(T_{1}\right)$.

Myocardial IR impairs systolic and diastolic function of left ventricle and causes a series of hemodynamic changes, such as decrease of myocardial contractility and compliance, discordance of myocardial contraction, increase of left ventricular end-diastolic pressure and reduction of stroke volume [5]. LVDP was calculated as LVSP minus left ventricular end diastolic pressure, and reflected left ventricular contractile function. The higher LVDP is, the better the left ventricular contractility is. Because it is an important variable of ventricular contractility, some researchers even selected LVDP as the only indicator to investigate contractile function in isolated perfused heart model [9] [10]. This study showed that LVDP significantly decreased after reperfusion, suggesting myocardial IR could indeed impair cardiac systolic function, even under the protection by cardioplegic solution. Importantly, this study demonstrated that the recovery of LVDP was higher in GC group than in IR group after reperfusion. This suggested that gradual clamping could reduce systolic dysfunction of left ventricle induced by myocardial IR.

LVSP and $+\mathrm{dp} / \mathrm{dt}_{\max }$ also reflect left ventricular contractile function, and $-\mathrm{dp} / \mathrm{dt}_{\max }$ reflects myocardial diastolic function, but in this study the recovery of LVSP, $+\mathrm{dp} / \mathrm{dt}_{\max }$ and $-\mathrm{dp} / \mathrm{dt}_{\max }$ between GC group and IR group could not reach statistical significance. However, the recovery curves of LVSP, $+\mathrm{dp} / \mathrm{dt}_{\max }$ and $-\mathrm{dp} / \mathrm{dt}_{\max }$ in GC group were all above the curves in IR group in Figures 2-4 respectively. Although differences in two groups could not reach statistical significance, all the $P$ values were relatively small (all the $P<0.20$ ). The relatively less sensitive of these variables in reflecting cardiac function and the relative small sample size in this study may contribute to the negative results.

LVDP was even higher than that at baseline in GC group in Figure 1, this may be due to two reasons. The hearts were excised from the rats and then connected to the Langendorff apparatus, although we controlled this process not exceeding 2 minutes, there did exist a period of global ischemia. Although the equilibrium time in some experiments was less than 30 minutes [9] [11], we are still wandering that 30 minutes of equilibrium may be not enough for the myocardial recovery, this need further investigation. On the other hand, gradual clamping could improve the recovery of contractile function after reperfusion.

There are few studies look similar like the present study, but actually focal points of these studies were different from us. Abrupt occlusion of coronary artery results in an immediate stop in myocardial perfusion, this is associated with a rapid change of metabolism such as a rapid fall in tissue levels of phosphocreatine and ATP [12] [13]. It has been shown that this down-regulation process in metabolism may be time-dependent [14]-[16]. If blood flow of the left anterior descending coronary artery was reduced gradually to the same level for moderate ischemia in the heart, Arai et al. found that the changes in these metabolic variables of ischemia were blunted compared to abrupt reduction of the blood flow [16]. Earlier studies have shown that graded reperfusion following ischemia, at flow rates below baseline, improved post-ischemic function compared with immediate reperfusion at baseline levels [17] [18]. Takeo et al. demonstrated that reperfusion with reduced flow rates following global ischemia could improve post-ischemic function compared to immediate reperfusion with normal flow rates [18]. Klawitter et al. found a period of low flow perfusion following global ischemia before full reperfusion improved post-ischemic myocardial function and energetic recovery [19]. These studies doubtlessly had important scientific sense. However, all these studies were designed based on a phenomenon and found another phenomenon, and investigated an isolated procedure for myocardial protection. The present study based on a novel systemic hypothesis, and it not only found a novel myocardial protection strategy, but more impor- 
tantly it verified the novel hypothesis of gradual adaptation through myocardial protection.

This study was also designed by a concept different from IPC. IPC, which is achieved by short episodes of IR applied before the lethal ischemia, has been shown to be an effective adjunct to myocardial protection in cardiac surgery [20]-[22]. However, IPC with repeated clamping of the aorta will never get widespread use [23]. Gradual clamping that we report here does not need repeated clamping with severe complications such as the aorta rupture, but only need an equipment to control the clamping rate without extra mechanical trauma. Therefore, gradual clamping has more potential of clinical application.

The isolated perfused mammalian heart model was established in 1897 by Oscar Langendorff. The simplicity of the isolated mammalian heart preparation makes this model high reproducibility and relatively low cost, and a broad spectrum of measurements can be done by using this apparatus [24]. Meanwhile, with the absence of normal humoral influences and neuronal regulation, the isolated perfused mammalian heart model directly reflects the impact of the experimental treatment on the heart. In the last decade the method has been widely used in many areas, especially in researches about myocardial IR injury [25]. This study also used this common model to explore the protective effects of gradual clamping and obtained reliable results. St Thomas' II cardioplegic solution was widely used in the clinic for cardiac surgery for myocardial protection. This solution was used to induce cardioplegia during global ischemia in this study, making the result of this study more reliable.

The major limitation of the study was that we did not further investigate the mechanism underlying gradual clamping. The myocardium rapidly adapted its own innate ongoing normal process to accommodate global ischemia during the period of gradual clamping. This adaptation resulted in functional improvements compared with hearts suffered from sudden-onset ischemia. Finding the substances mediating this adaptation is also an important goal in our future work. Since the protective effect in this study occurred in the absence of prior reperfusion, whether the processes known to be stimulated during reperfusion, such as oxygen-derived free radical release [26], has contribution to the present result need to be further explored.

These findings may provide a new direction for the myocardial protection or other organs' protection. Because of its potential clinical value, the in vivo study need to be carried out and the related mechanism need to be explored.

\section{Conclusion}

This is the first study showing that gradual clamping can improve recovery of systolic function of left ventricle after myocardial IR. This initially verified our gradual adaptation hypothesis.

\section{Acknowledgements}

We thank Baofeng Lv, Department of Anesthesiology, First Affiliated Hospital, Zhengzhou University, for taking part in the experiment. We thank Wanjun Zheng, Department of Anesthesia, First Affiliated Hospital of Fujian Medical University, for helping the draft writing. We thank the associate professor Jinxin Zhang, Department of Medical Statistics and Epidemiology, School of Public Health in Sun Yat-sen University (Guangzhou, China) for providing guidance on experimental design and statistical analysis.

\section{Sources of Grants}

This work was supported by a grant from the Department of science and technology of Guangdong Province (No. 2010B060900101).

\section{Declaration of Interests}

All authors have no interests to be declared.

\section{Note}

The abstract of this paper was accepted for ePoster discussion at the 2016 WCA (16 $6^{\text {th }}$ World Congress of Anaesthesiologists ) will be held in August at Hong Kong.

\section{References}

[1] Hessel, E.N. (2014) A Brief History of Cardiopulmonary Bypass. Seminars in Cardiothoracic and Vascular Anesthesia, 18, 87-100. http://dx.doi.org/10.1177/1089253214530045 
[2] Khan, N.E., De Souza, A., Mister, R., Flather, M., Clague, J., Davies, S., Collins, P., Wang, D., Sigwart, U. and Pepper, J. (2004) A Randomized Comparison of Off-Pump and On-Pump Multivessel Coronary-Artery Bypass Surgery. The New England Journal of Medicine, 350, 21-28. http://dx.doi.org/10.1056/NEJMoa031282

[3] Nathoe, H.M., van Dijk, D., Jansen, E.W., et al. (2003) A Comparison of On-Pump and Off-Pump Coronary Bypass Surgery in Low-Risk Patients. The New England Journal of Medicine, 348, 394-402. http://dx.doi.org/10.1056/NEJMoa021775

[4] Xiong, J., Liao, X., Xue, F.S., Yuan, Y.J., Wang, Q. and Liu, J.H. (2011) Remote Ischemia Conditioning-an Endogenous Cardioprotective Strategy from Outside the Heart. Chinese Medical Journal (English), 124, 2209-2215.

[5] Sanada, S., Komuro, I. and Kitakaze, M. (2011) Pathophysiology of Myocardial Reperfusion Injury: Preconditioning, Postconditioning, and Translational Aspects of Protective Measures. American Journal of Physiology—Heart and Circulatory Physiology, 301, H1723-H1741. http://dx.doi.org/10.1152/ajpheart.00553.2011

[6] Kolh, P. (2014) Remote Ischaemic Pre-Conditioning in Cardiac Surgery: Benefit or Not? European Heart Journal, 35, 141-143. http://dx.doi.org/10.1093/eurheartj/eht517

[7] Kharbanda, R.K., Nielsen, T.T. and Redington, A.N. (2009) Translation of Remote Ischaemic Preconditioning into Clinical Practice. Lancet, 374, 1557-1565. http://dx.doi.org/10.1016/S0140-6736(09)61421-5

[8] Ludman, A.J., Yellon, D.M. and Hausenloy, D.J. (2010) Cardiac Preconditioning for Ischaemia: Lost in Translation. Disease Models \& Mechanisms, 3, 35-38. http://dx.doi.org/10.1242/dmm.003855

[9] Hendgen-Cotta, U.B., Merx, M.W., Shiva, S., et al. (2008) Nitrite Reductase Activity of Myoglobin Regulates Respiration and Cellular Viability in Myocardial Ischemia-Reperfusion Injury. Proceedings of the National Academy of Sciences of the United States of America, 105, 10256-10261. http://dx.doi.org/10.1073/pnas.0801336105

[10] Seubert, J., Yang, B., Bradbury, J.A., et al. (2004) Enhanced Postischemic Functional Recovery in CYP2J2 Transgenic Hearts Involves Mitochondrial ATP-Sensitive K+ Channels and p42/p44 MAPK Pathway. Circulation Research, 95, 506-514. http://dx.doi.org/10.1161/01.RES.0000139436.89654.c8

[11] Allan, A., Fenning, A., Levick, S., Hoey, A. and Brown, L. (2005) Reversal of Cardiac Dysfunction by Selective ET-A Receptor Antagonism. British Journal of Pharmacology, 146, 846-853. http://dx.doi.org/10.1038/sj.bjp.0706384

[12] Jennings, R.B., Murry, C.E., Steenbergen, C.J. and Reimer, K.A. (1990) Development of Cell Injury in Sustained Acute Ischemia. Circulation, 82, I2-I12.

[13] Schaefer, S., Schwartz, G.G., Wisneski, J.A., et al. (1992) Response of High-Energy Phosphates and Lactate Release during Prolonged Regional Ischemia in Vivo. Circulation, 85, 342-349. http://dx.doi.org/10.1161/01.CIR.85.1.342

[14] Pantely, G.A., Malone, S.A., Rhen, W.S., Anselone, C.G., Arai, A., Bristow, J. and Bristow, J.D. (1990) Regeneration of Myocardial Phosphocreatine in Pigs Despite Continued Moderate Ischemia. Circulation Research, 67, 1481-1493. http://dx.doi.org/10.1161/01.RES.67.6.1481

[15] Arai, A.E., Pantely, G.A., Anselone, C.G., Bristow, J. and Bristow, J.D. (1991) Active Downregulation of Myocardial Energy Requirements during Prolonged Moderate Ischemia in Swine. Circulation Research, 69, 1458-1469. http://dx.doi.org/10.1161/01.RES.69.6.1458

[16] Arai, A.E., Grauer, S.E., Anselone, C.G., Pantely, G.A. and Bristow, J.D. (1995) Metabolic Adaptation to a Gradual Reduction in Myocardial Blood Flow. Circulation, 92, 244-252. http://dx.doi.org/10.1161/01.CIR.92.2.244

[17] Matsubara, T. (1990) Beneficial Effects of Low-Flow Perfusion Resumed Early after Zero-Flow Ischemia on Myocardial Energy Metabolism and Mechanical Function: 31P-NMR Study in the Isolated Perfused Rat Heart. The Tohoku Journal of Experimental Medicine, 161, 241-250. http://dx.doi.org/10.1620/tjem.161.241

[18] Takeo, S., Liu, J.X., Tanonaka, K., Nasa, Y., Yabe, K., Tanahashi, H. and Sudo, H. (1995) Reperfusion at Reduced Flow Rates Enhances Postischemic Contractile Recovery of Perfused Heart. American Journal of Physiology, 268, H2384-H2395.

[19] Klawitter, P.F., Murray, H.N., Clanton, T.L., Palmer, B.S. and Angelos, M.G. (2002) Low Flow after Global Ischemia to Improve Postischemic Myocardial Function and Bioenergetics. Critical Care Medicine, 30, 2542-2547. http://dx.doi.org/10.1097/00003246-200211000-00023

[20] Ji, B.Y., Liu, M.Z., Liu, J.P., Wang, G.Y., Feng, W., Lu, F. and Hu, S.S. (2007) Evaluation by Cardiac Troponin I: The Effect of Ischemic Preconditioning as an Adjunct to Intermittent Blood Cardioplegia on Coronary Artery Bypass Grafting. Journal of Cardiac Surgery, 22, 394-400. http://dx.doi.org/10.1111/j.1540-8191.2007.00433.x

[21] Codispoti, M., Sundaramoorthi, T., Saad, R.A., Reid, A., Sinclair, C. and Mankad, P. (2006) Optimal Myocardial Protection Strategy for Coronary Artery Bypass Grafting without Cardioplegia: Prospective Randomised Trial. Interactive cardiovascular and Thoracic Surgery, 5, 217-221. http://dx.doi.org/10.1510/icvts.2005.116863

[22] Zhang, J.Q., Wang, Q., Xue, F.S., Li, R.P., Cheng, Y., Cui, X.L., Liao, X. and Meng, F.M. (2013) Ischemic Preconditioning Produces More Powerful Anti-Inflammatory and Cardioprotective Effects than Limb Remote Ischemic Post- 
conditioning in Rats with Myocardial Ischemia-Reperfusion Injury. Chinese Medical Journal (English), 126, 39493955.

[23] Vaage, J. and Valen, G. (2000) Preconditioning and Cardiac Surgery. The Annals of Thoracic Surgery, 75, S709-S714. http://dx.doi.org/10.1016/S0003-4975(02)04696-9

[24] Ytrehus, K. (2000) The Ischemic Heart-Experimental Models. Pharmacological Research, 42, 193-203. http://dx.doi.org/10.1006/phrs.2000.0669

[25] Skrzypiec-Spring, M., Grotthus, B., Szelag, A. and Schulz, R. (2007) Isolated Heart Perfusion According to Langendorff-Still Viable in the New Millennium. Journal of Pharmacological and Toxicological Methods, 55, 113-126. http://dx.doi.org/10.1016/j.vascn.2006.05.006

[26] Zweier, J.L., Flaherty, J.T. and Weisfeldt, M.L. (1987) Direct Measurement of Free Radical Generation Following Reperfusion of Ischemic Myocardium. Proceedings of the National Academy of Sciences of the United States of America, 84, 1404-1407. http://dx.doi.org/10.1073/pnas.84.5.1404 